\title{
Intra-amygdala infusion of zeta inhibitory peptide attenuates neuropathic pain but not inflammatory pain in adult rats
}

\author{
Juan Yao $^{1}$, Ling Zhang ${ }^{2}$, Weiping Lei $^{3}$, Yaqin Huang ${ }^{3}$, Honghai Zhang ${ }^{3}$, Bo Lu ${ }^{4}$, Yongxing Yao ${ }^{5}$, \\ Li Zhao ${ }^{6}$, Jianliang Sun ${ }^{2}$
}

${ }^{1}$ Department of Anesthesia, Zhongda Hospital affiliated to Southeast University, Nanjing 210009, China; ${ }^{2}$ The Fourth Clinical Medical College of Zhejiang Chinese Medical University, Hangzhou 310003, China; ${ }^{3}$ Department of Anesthesia, Hangzhou First People's Hospital Affiliated to Zhejiang University school of Medicine, Hangzhou 310003, China; ${ }^{4}$ Department of Anesthesia, Ningbo Huamei Hospital, University of Chinese Academy of Sciences, Ningbo 315000, China; ${ }^{5}$ Department of Anesthesia, First Affiliated Hospital, ${ }^{6}$ Department of Anesthesia, Second Affiliated Hospital, Zhejiang University School of Medicine, Hangzhou 310009, China

Contributions: (I) Conception and design: J Yao, L Zhang, J Sun; (II) Administrative support: J Sun; (III) Provision of study materials: W Lei, Y Huang, H Zhang; (IV) Collection and assembly of data: J Yao, B Lu, Y Yao; (V) Data analysis and interpretation: J Yao, L Zhao; (VI) Manuscript writing: All authors; (VII) Final approval of manuscript: All authors.

Correspondence to: Jianliang Sun, MD. The Fourth Clinical Medical College of Zhejiang Chinese Medical University, Hangzhou 310003, China. Email: jxmzsj1@163.com.

Background: Protein kinase $\mathrm{M} \zeta(\mathrm{PKM} \zeta)$, a typical brain-specific PKC isoform, has been shown to be critical in the maintenance of long-term potentiation and memory storage. Zeta inhibitory peptide (ZIP), a peptide with selective inhibition of $\mathrm{PKM} \zeta$, has been used in relieving experimental neuropathic pain and disrupting memory. The aim of this study was to investigate the effects of intra-amygdalar infusion of ZIP on neuropathic pain induced by chronic constriction injury (CCI), and inflammatory pain induced by complete Freund's adjuvant (CFA) in adult rats. Methods: (I) ZIP was infused into the amygdala 30 minutes (min) before CCI was performed. Mechanical withdrawal threshold (MWT) was determined prior to the infusion of ZIP, and 30, 60, 90, 120, 150, $180 \mathrm{~min}$ after CCI (n=8 per group). (II) ZIP was infused into the amygdala 3, 7, 14 days after CCI (n=8 per group). MWT was measured 30 min before the infusion and 2, 12, $24 \mathrm{~h}$ after the infusion. (III) Three days after CCI, ZIP was infused into the amygdala repeatedly once a day for 2 days. MWT was measured before each infusion and 2 or $24 \mathrm{~h}$ after each infusion ( $\mathrm{n}=8$ per group). (IV) ZIP was infused into the amygdala $24 \mathrm{~h}$ after the establishment of inflammatory pain induced by complete Freund's adjuvant (CFA). MWT was determined $30 \mathrm{~min}$ before the infusion and 30, 60, 90, 120 and $150 \mathrm{~min}$ after the infusion (n=8 per group).

Results: As shown in figures, (I) amygdalar infusion of ZIP prior to the CCI produced no effect on CCIinduced hyperalgesia when compared to scr-ZIP or saline infusion $[\mathrm{n}=8$, interaction effect, $\mathrm{F}(7.312,76.776)$ $=1.237, \mathrm{P}>0.05$; time as main factor, $\mathrm{F}(3.656,76.776)=115.346, \mathrm{P}<0.001$; group as main factor, $\mathrm{F}(2,21)=0.648$, $\mathrm{P}>0.05]$. (II) BLA infusion of ZIP significantly increased mechanical withdrawal threshold 7 days after CCI [n=8, interaction effect, $\mathrm{F}(5.476,57.500)=15.279, \mathrm{P}<0.001$; time as main factor, $\mathrm{F}(2.738,57.500)=242.357, \mathrm{P}<0.001$; group as main factor, $\mathrm{F}(2,21)=4.786, \mathrm{P}<0.05]$, just the same as 3 and 14 days after CCI (data not shown). (III) Bilateral injection of ZIP into the BLA significantly reduced mechanical hyperalgesia $2 \mathrm{~h}$ after the administration $[\mathrm{n}=8$, paired t-test, $\mathrm{t}(0.05,7)=-5.561, \mathrm{P}<0.05 ; \mathrm{n}=8$, paired t-test, $\mathrm{t}(0.05,7)=-4.745, \mathrm{P}<0.05]$, and returned to baseline $24 \mathrm{~h}$ after the administration $[\mathrm{n}=8$, paired t-test, $\mathrm{t}(0.05,7)=1.039, \mathrm{P}>0.05 ; \mathrm{n}=8$, paired t-test, $\mathrm{t}(0.05,7)$ $=-1.173, \mathrm{P}>0.05]$. (IV) ZIP produced no effect on mechanical withdrawal thresholds at all time points examined after the infusion, compared with scr-ZIP or saline control groups $(\mathrm{n}=8$, interaction effect, $\mathrm{F}(6.135,126)=1.724$, $\mathrm{P}>0.05$; group as main factor, $\mathrm{F}(2,21)=0.608, \mathrm{P}>0.05)$.

Conclusions: Intra-amygdala infusion of ZIP attenuates mechanical hyperalgesia induced by CCI but has no effect on inflammatory pain induced by CFA in rats, suggesting that amygdala PKM $\zeta$ may be a therapeutic target in the treatment of neuropathic pain.

Keywords: Amygdala; protein kinase $\mathrm{M \zeta}(\mathrm{PKM} \zeta)$; zeta inhibitory peptide (ZIP); neuropathic pain; inflammatory pain 
Submitted Sep 18, 2019. Accepted for publication Nov 18, 2019.

doi: 10.21037/apm.2019.11.21

View this article at: http://dx.doi.org/10.21037/apm.2019.11.21

\section{Introduction}

Pain and memory may share some common neurological pathways, as shown by the fact that alleviation of pain has been related to memory loss in patients (1). The accepted concept is that neuroplasticity can lead to the development of chronic pain memory, and the engram of pain may contribute to the persistence of a chronic pain state even after the trauma has been resolved. Therefore, for pain therapy development, it is critical to reveal molecular mechanisms underlying the maintenance of synaptic plasticity in the development of pain and memory. The physiological basis of synaptic plasticity most commonly studied in mammalian systems is long-term potentiation (LTP), a synaptic substrate for learning and memory (2). PKM $\zeta$ has been found to participate in the maintenance of LTP, which is critical for memory storage (3). Previous findings showed that blocking $\mathrm{PKM} \zeta$ activity by zeta inhibitory peptide (ZIP), a myristoylated $\zeta$-pseudosubstrate peptide, will disrupt some stored memories, including spatial memory in the hippocampus, aversive memories, and foreground context fear memory in the basolateral amygdala, appetitive memories in the nucleus accumbens, and skilled sensorimotor memories in the neocortex (4-6). All these findings indicate that ZIP might disrupt amygdaladependent memory.

The basolateral nucleus of the amygdala (BLA), a part of the limbic regions, is a key brain region for the modulation of emotion and pain (7). BLA lesion with ibotenic acid before the establishment of neuropathic pain attenuated mechanical sensitivity induced by spared nerve injury in rats, indicating that the BLA activities may be important for the development and maintenance of pain after nerve damage, and pain-related memories and amygdala-related negative emotions could lead to pain chronicity (8). The above findings suggest that the amygdala is associated with pain-related negative emotional response and memory formation. Therefore, it is helpful to understand the processes involved in forming pain-related emotional responses and painful memories in order to understand the integration mechanism of pain signals in the amygdala.

Previous studies showed that ZIP administration into the anterior cingulate cortex alleviated mechanical hyperalgesia induced by chronic constriction injury (CCI) of the sciatic nerve, whereas infusion of ZIP into the subarachnoid space reduced the mechanical hyperalgesia induced by intraplantar injection of complete Freund's adjuvant (CFA) $(9,10)$, however, there is no data that exists about the effects of amygdalar infusion of ZIP on painrelated behavior. Here we investigated the effect of ZIP on CCI-induced neuropathic pain memory and observed the effects of ZIP on the development of inflammatory pain induced by CFA.

\section{Methods}

\section{Animals}

Male Sprague-Dawley rats, weighing 220-300 grams at the time of testing, were obtained from the Laboratory Animal Center, Academy of Medical Sciences of Zhejiang Province. They were housed in temperature $\left(24 \pm 2{ }^{\circ} \mathrm{C}\right)$ and humidity $(50 \% \pm 5 \%)$-controlled room on a 12 hours (h) light/dark cycle, where food and water were freely available. All procedures were approved by the experimental animal ethics committee of Wenzhou Medical University (wydw2012-0095) and consistent with the ethical guidelines for investigation of experimental pain in animals.

\section{Experimental protocols}

We used four experimental protocols: (1) ZIP was infused into the amygdala 30 minutes $(\mathrm{min})$ before CCI was performed. Mechanical withdrawal threshold (MWT) was determined prior to the infusion of ZIP, and 30, 60, 90, 120, 150, $180 \mathrm{~min}$ after CCI ( $\mathrm{n}=8$ per group). (2) ZIP was infused into the amygdala 3, 7, 14 days after CCI ( $\mathrm{n}=8$ per group). MWT was measured 30 min before the infusion and 2, 12, $24 \mathrm{~h}$ after the infusion (3). Three days after CCI, ZIP was infused into the amygdala repeatedly once a day for 2 days. MWT was measured before each infusion and 2 or $24 \mathrm{~h}$ after each infusion ( $\mathrm{n}=8$ per group) (4). ZIP was infused into the amygdala $24 \mathrm{~h}$ after the establishment of inflammatory pain induced by complete Freund's adjuvant (CFA). MWT was determined $30 \mathrm{~min}$ before the infusion and 30, 60, 90, 120 and $150 \mathrm{~min}$ after the infusion ( $\mathrm{n}=8$ per group). 


\section{Establishment of pain models}

\section{Loose sciatic nerve ligation}

Peripheral neuropathic pain was induced by chronic constriction injury (CCI) in rats, according to Bennett and $\mathrm{Xie}$ (11). The rats were anesthetized with an intraperitoneal injection of $4 \%$ chloral hydrate $(400 \mathrm{mg} / \mathrm{kg})$. After the corneal reflex and righting reflex disappeared, the rats were fixed on the operating table. The hair around the mid-thigh was shaved, and the skin was sterilized with povidone-iodine. Blunt dissection was made through the biceps femoris, and proximal to the sciatic trifurcation, the common sciatic nerve of the left hind limb was exposed at the level of the middle of the thigh and then ligatured by four loops with 4-0 silk, $1 \mathrm{~mm}$ apart. We noted that the thigh muscle twitched when the sciatic nerve was ligatured, indicating that the ligation of the silk was not too tight or too loose. Muscular and skin layers were sutured, and the povidone-iodine solution was embrocated externally. For additional rats that served as sham CCI, the left sciatic nerve was exposed but not ligated.

\section{CFA-induced chronic inflammatory pain model}

Rats were anesthetized with isoflurane in $100 \% \mathrm{O}_{2}$ (3\% for induction, $2 \%$ for maintenance), and the plantar surface of the left hind paw was injected with $100 \mu \mathrm{L}$ CFA (50\%; Sigma, St. Louis, MO) to establish a chronic inflammatory pain model. Twenty-four hours after CFA injection, typical peripheral inflammatory symptoms appeared. The same volume of normal saline was administered in the sham controls.

\section{Placement and verification of bilateral amygdala cannulation}

Rats were anesthetized with an intraperitoneal (i.p.) injection of $4 \%$ chloral hydrate $(400 \mathrm{mg} / \mathrm{kg})$, and bilateral amygdalar cannulation was performed as described previously $(5,12)$. Two 26-gauge guide cannulas were aimed at the BLA using stereotaxic coordinates $(3 \mathrm{~mm}$ posterior, $\pm 4.8 \mathrm{~mm}$ lateral, $8.6 \mathrm{~mm}$ ventral) relative to the bregma (13). Guide cannulas were cemented in place and secured to the skull with stainless steel screws, superglue, and dental acrylic. For all study subjects, stainless steel dummy cannulas were inserted in the internal cannulae when rats were not in use, to prevent occlusion until drug delivery. Following the surgery, the incision was swabbed with lidocaine $(2 \%)$ to minimize discomfort during the recovery period. Each rat received penicillin and could recover for 7 days prior to any behavioral tests or surgery. No signs of weight loss or distress were observed after surgery.

After behavioral tests, $4 \%$ methylene blue $(0.5 \mu \mathrm{L} /$ side $)$ was injected by an internal cannula to stain the injection site and observe the drug diffusion range. The rats were killed by an i.p. overdose of $4 \%$ chloral hydrate and perfused with $300 \mathrm{~mL}$ normal saline, followed by $300 \mathrm{~mL} 4 \%$ paraformaldehyde. Brains were then removed, and lumps of tissue containing the amygdalae were harvested and postfixed in $4 \%$ paraformaldehyde $\left(4^{\circ} \mathrm{C}\right)$ for $4-6 \mathrm{~h}$. The tissue was dehydrated in $30 \%$ sucrose for $24 \mathrm{~h}$ and frozen $30 \mu \mathrm{m}$ coronal sections were prepared. The location of methylene blue was recorded according to a rat brain atlas (13). We further verified the cannula placement under a microscope by observing the location of the cannula tip in one slice with hematoxylin and eosin staining. Rats with cannulas outside the amygdala were excluded in the final data analyses.

\section{Administration of ZIP}

ZIP (Tocris Bioscience, Britain) and scrambled (scr) ZIP (Tocris Bioscience, Britain), or normal saline $(10 \mathrm{nmoL} / \mu \mathrm{L}$, $0.5 \mu \mathrm{L} /$ side, $\mathrm{n}=8$ per group), was injected into the basolateral amygdala with a $1 \mu \mathrm{L}$ microsyringe attached to an additional cannulae, which was in excess of the guide cannulae by approximately $0.5-0.7 \mathrm{~mm}$, over a period of $60 \mathrm{~s}$. ZIP and the inactive version of ZIP (scr-ZIP) were dissolved in normal saline (as a vehicle). Rats injected with vehicle were used as a control group. After the injection, the syringe was left in place for an additional 90 seconds (s) to ensure adequate diffusion. The dose and volume of the agents were chosen based on previous studies to allow coverage throughout the amygdala $(5,12,14)$. Immediately following the infusion, rats were returned to their individual home cages.

\section{Bebavioral test for mechanical byperalgesia}

To avoid any variability in behavioral responses that might be induced by environmental influences, the rats were placed into a quiet room, and all experiments were performed by a blinded operator. One day after CFA injection, or 3, 7, 14 days after sciatic nerve ligation, rats were placed into a plastic chamber $(20 \mathrm{~cm} \times 20 \mathrm{~cm} \times 30 \mathrm{~cm})$, which was positioned on the top of foraminiferous barbed wire, thus making it convenient to observe the mechanical stimuli to the plantar surface of both hind paws. The rats could adapt and habituate to the surrounding environment for 


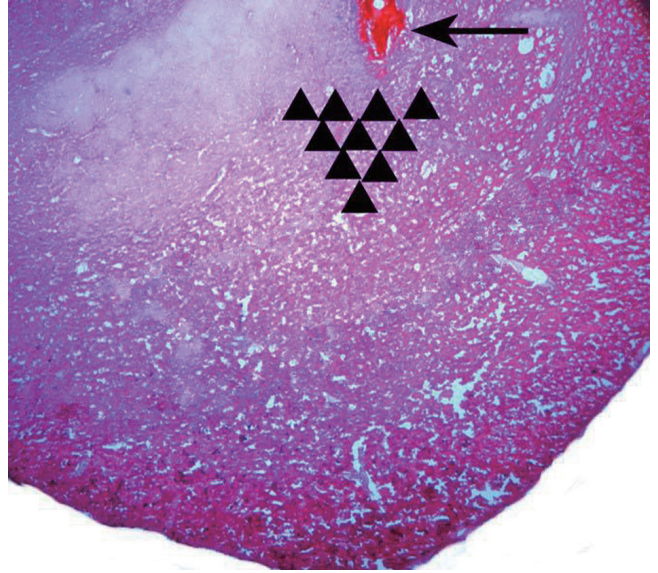

Figure 1 A micrograph $(40 x)$ showing the infusion site in the BLA. The picture shows the location of the cannula tip in one amygdala coronal slice. The black arrows represent the location of the cannula tip. BLA, basolateral nucleus of the amygdala.

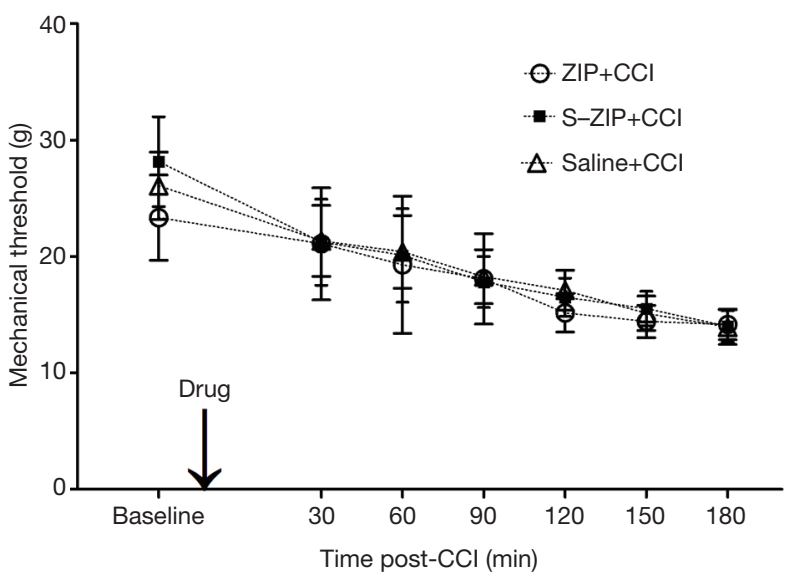

Figure 2 The effects of preventive ZIP on CCI induced hyperalgesia. Bilateral microinjections of ZIP into BLA had no effect on mechanical withdrawal thresholds when compared to scrZIP or saline rats. All data are presented as means \pm SEM, $\mathrm{P}>0.05$. ZIP, zeta inhibitory peptide; CCI, chronic constriction injury; BLA, basolateral nucleus of the amygdala.

15 min, and then mechanical threshold measurements for each hind paw were obtained using a mechanical, electronic stimulator (BME-404, Biomedical Engineering Institute of Chinese Academy of Medical Sciences, China) in response to probing of the plantar skin. Mechanical pressure was applied with a $0.6 \mathrm{~mm}$ filament (force, $0.1 \mathrm{~g}$ ) to test the mechanical hyperalgesia. For each measurement, pressure was applied 6 times with an inter-trial interval of $4 \mathrm{~s}$.
If there was a response to the stimuli of the cellosilk, the rats raised or licked their feet. Otherwise, it was recorded as a negative response to the stimuli, and the maximum stimulus was $50 \mathrm{~g}$. The testing was repeated 3 times, and the values were averaged. The results were analyzed using a Dixon nonparametric test, as previously described $(15,16)$.

\section{Statistical analysis}

Data were expressed as mean \pm standard error of means (SEM). Two data sets were compared with a Student's $t$-test (paired or unpaired when appropriate). The statistical analyses were performed using two-way analysis of variance with time point as repeated measures (two-way mixedmodel ANOVA) followed by Bonferroni's post-test, using SPSS17.0, and the statistical figures were all performed using Graph Pad Prism 5.0 software. For all analyses, $\mathrm{P}<0.05$ was considered statistically significant.

\section{Results}

Most of the infusion sites were found to be correctly administered or just above the BLA (Figure 1). However, five animals were excluded from analysis due to misplaced cannulas.

\section{ZIP infusion prior to nerve injury produced no effect on mechanical byperalgesia induced by CCI}

To investigate the preventive effect of amygdalar ZIP infusion on mechanical hyperalgesia induced by CCI, we delivered ZIP into the amygdala and then ligated the sciatic nerve. MWT was measured before and 30, 60, 90, 120, 150, 180 min after nerve injury. As shown in Figure 2, amygdalar infusion of ZIP prior to the CCI produced no effect on CCI-induced hyperalgesia when compared to scr-ZIP or saline infusion $[\mathrm{n}=8$, interaction effect, $\mathrm{F}(7.312,76.776)$ $=1.237, \mathrm{P}>0.05$; time as main factor, $\mathrm{F}(3.656,76.776)$ $=115.346, \mathrm{P}<0.001$; group as main factor, $\mathrm{F}(2,21)=0.648$, $\mathrm{P}>0.05]$. These results indicated that amygdalar infusion of ZIP prior to the CCI did not disrupt the pain sensitivity induced by CCI.

\section{ZIP infusion after nerve injury attenuates mechanical byperalgesia induced by CCI}

We next investigated whether BLA infusion of ZIP could alleviate CCI-induced hyperalgesia at distinct stages after 


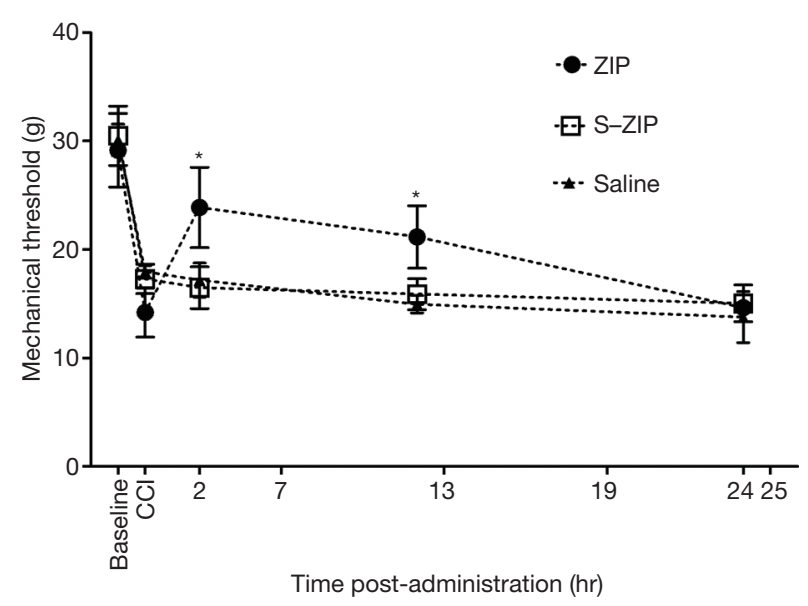

Figure 3 Effects of ZIP infusion on CCI-induced mechanical hyperalgesia. Bilateral microinjections of ZIP into the BLA decreased mechanical hyperalgesia 7 days after CCI, compared to scr-ZIP or saline, $\mathrm{P}<0.05$. Data are expressed as means $\pm \mathrm{SEM}$, $\mathrm{n}=8$. ZIP, zeta inhibitory peptide; CCI, chronic constriction injury.

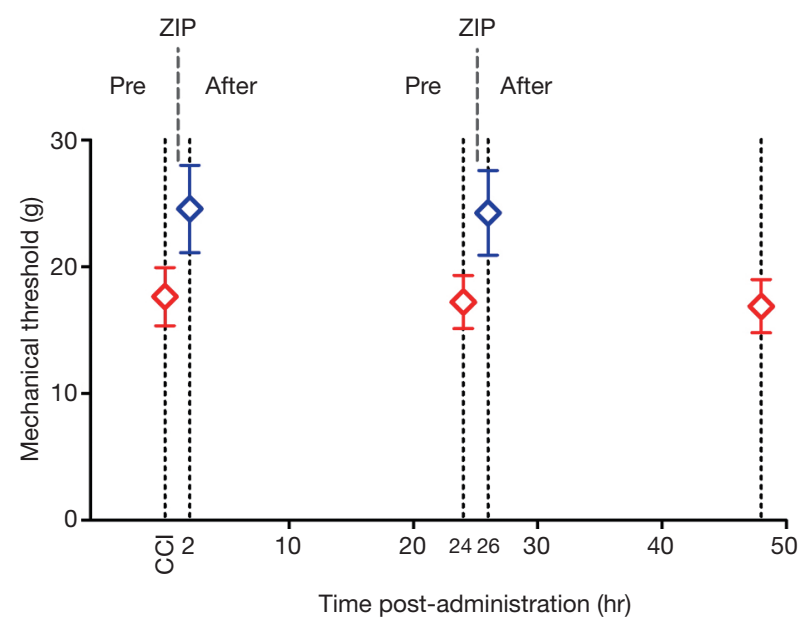

Figure 4 Repetitive amygdalar infusion of ZIP on CCI induced hyperalgesia. The MWTS were measured before drug administration as baseline and 2 or $24 \mathrm{~h}$ after ZIP infusion. Bilateral microinjections of ZIP into BLA decreased hyperalgesia induced by CCI at $2 \mathrm{~h}$ after drug administration, compared to baseline. Data are expressed as means \pm SEM, $\mathrm{n}=8, \mathrm{P}<0.05$. ZIP, zeta inhibitory peptide; CCI, chronic constriction injury; BLA, basolateral nucleus of the amygdala.

nerve injury. MWT was measured 2, 12, $24 \mathrm{~h}$ after drug administration. We found that compared to scr-ZIP or saline control groups, BLA infusion of ZIP significantly increased mechanical withdrawal threshold 7 days after
CCI $[\mathrm{n}=8$, interaction effect, $\mathrm{F}(5.476,57.500)=15.279$, $\mathrm{P}<0.001$; time as main factor, $\mathrm{F}(2.738,57.500)=242.357$, $\mathrm{P}<0.001$; group as main factor, $\mathrm{F}(2,21)=4.786, \mathrm{P}<0.05$; Figure 3], just the same as 3 and 14 days after CCI (data not shown). These results suggest that ZIP infusion alleviates mechanical hyperalgesia induced by CCI.

\section{Repetitive amygdalar infusion of ZIP produced similar effects as a single dose}

The purpose of this experiment was to further investigate the effect of ZIP on neuropathic pain by repetitive administration of ZIP after related-pain memory recovery. MWT was tested 2 and $24 \mathrm{~h}$ following the infusion, and then a second dose was given. Bilateral injection of ZIP into the BLA significantly reduced mechanical hyperalgesia $2 \mathrm{~h}$ after the administration [Figure 4; $\mathrm{n}=8$, paired $t$-test, $t(0.05,7)=-5.561, \mathrm{P}<0.05 ; \mathrm{n}=8$, paired $t$-test, $t(0.05,7)$ $=-4.745, \mathrm{P}<0.05)$, and returned to baseline $24 \mathrm{~h}$ after the administration (Figure $4 ; \mathrm{n}=8$, paired $t$-test, $t(0.05,7)$ $=1.039, \mathrm{P}>0.05 ; \mathrm{n}=8$, paired $t$-test, $t(0.05,7)=-1.173$, $\mathrm{P}>0.05]$. This result indicated that the analgesic effect of ZIP lasted for more than $2 \mathrm{~h}$. The second infusion produced a similar effect as the first dose, suggesting that ZIP may transiently disrupt, but not erase the expression of painrelated memory (Figure 3).

\section{BLA microinjection of ZIP did not attenuate the inflammatory pain induced by intraplantar CFA in rats}

Mechanisms underlying neuropathic and inflammatory pain may differ. We, therefore, used a CFA-induced chronic inflammatory pain model to test the effect of amygdalar infusion of ZIP on inflammatory pain in rats. $24 \mathrm{~h}$ after CFA administration, ZIP was infused into the BLA. MWT was measured at 30, 60, 90, 120, and $150 \mathrm{~min}$ after ZIP infusion. As shown in Figure 5, ZIP produced no effect on mechanical withdrawal thresholds at all time points examined after the infusion, compared with scr-ZIP or saline control groups $[\mathrm{n}=8$, interaction effect, $\mathrm{F}(6.135,126)=1.724, \mathrm{P}>0.05$; group as main factor, $\mathrm{F}(2,21)=0.608, \mathrm{P}>0.05]$. These results suggest that BLA infusion of ZIP does not influence mechanical hyperalgesia induced by CFA.

\section{Discussion}

In this study, we observed three major phenomena: (I) ZIP infusion before pain-related memory formation did 


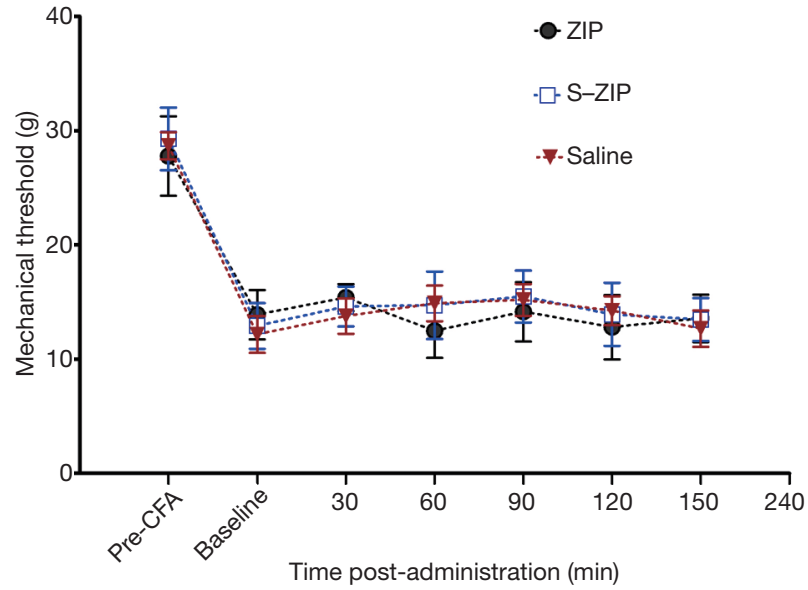

Figure 5 Effects of ZIP infusion on mechanical hyperalgesia induced by CFA. Twenty-four hours after CFA administration, ZIP was infused into the BLA. MWT was measured at 30, 60, 90, 120, and $150 \mathrm{~min}$ after drug infusion. Bilateral microinjections of ZIP into BLA had no effect on mechanical withdrawal threshold when compared to scr-ZIP or saline. All data are presented as means \pm SEM, P>0.05, n=8. ZIP, zeta inhibitory peptide; CCI, chronic constriction injury; CFA, complete Freund's adjuvant; BLA, basolateral nucleus of the amygdala.

not disrupt mechanical hyperalgesia; (II) ZIP disrupted pain-related memory induced by CCI but not CFA; (III) Repetitive ZIP infusion into the amygdala produced similar effects as a single dose.

The amygdala is responsible for painful memory, painrelated negative emotion, and avoidance behavior (17). Inhibition of $\mathrm{PKM} \zeta$ in the amygdala by a selective inhibitor ZIP, which consists of 13 auto-inhibitory amino acid pseudosubstrate from the regulatory domain of $\mathrm{PKM} \zeta$, leads to the erasure of foreground context fear memories, drug reward and aversion memory, and avoidance responses and conditioned taste-aversion. It has been suggested that the potential synaptic mechanism of ZIP is inhibition of $\mathrm{PKM} \zeta$ synthesis and prevention of LTP $(5,6,18)$. Therefore, we assumed that $\mathrm{PKM} \zeta$ might participate in amygdalarelated memory. PKM $\zeta$ has been previously shown to have importance in the maintenance and consolidation of long-term memory and LTP via upregulation of an NSF/ GluR2-dependent AMPAR trafficking pathway (19). To our knowledge, in this study we demonstrated for the first time that infusion of ZIP in the amygdala could attenuate mechanical hyperalgesia induced by CCI; however, ZIP was not able to alleviate CFA-induced inflammatory pain.

A previous study revealed that the anterior cingulate cortex (ACC), a key part of the limbic system, was responsible for processing the affective-emotional features of pain (20) and suggested that ZIP acted mechanistically by blocking PKM $\zeta$ activity (9). In contrast, more recently, it has been suggested that ZIP may lack specificity for PKM $\zeta$ inhibition and questioned the role of $\mathrm{PKM} \zeta$ as a memory maintenance molecule (21). Other mechanisms, including the hypothesis that ZIP reverses excitatory postsynaptic potential (EPSP) responses to disrupt memory, still could not be ruled out (22).

Our study suggested that ZIP was beneficial for neuropathic but not inflammatory pain. Although abnormal signal amplification in the central nervous system is a common mechanism underlying hyperalgesia following inflammation and nerve injury, the synaptic amplification for nociception is not identical between these two conditions. For example, inflammation-induced low-frequency afferent barrage triggers LTP in spino-periaqueductal gray (PAG) neurons, but high-level activity in nociceptive nerve fibers caused by nerve injury induces LTP in spino-parabrachial $(\mathrm{PB})$ neurons (23). A recent study has demonstrated that spinal administration of ZIP relieved hyperalgesia in inflammatory rats rather than CCI rats (10). Moreover, Li et al. (9) found that the infusion of ZIP into the ACC alleviated neuropathic pain. Taken together, these differential outcomes following ZIP administration indicate that persistent nociceptive processing in the amygdala has distinct molecular mechanisms between inflammatory and neuropathic pain. In addition, the phenomenon may be attributed to the amygdala receiving several types of afferent barrages. Further studies are necessary to confirm the differential roles of ZIP administration at different target sites during inflammatory and neuropathic pain development.

\section{Conclusions}

Our results show that intra-amygdalar infusion of ZIP attenuates mechanical hyperalgesia induced by CCI but has no effect on inflammatory pain induced by CFA in rats, suggesting that amygdala $\mathrm{PKM} \zeta$ may be a therapeutic target in the treatment of neuropathic pain.

\section{Acknowledgments}

Funding: This work was supported by the Natural Science Foundation of Zhejiang Province (Y17C09009) and the social development project of Hangzhou Science and Technology Commission (20170533B232). We are very grateful to Chun 
Xiao MD, $\mathrm{PhD}$, who provided his help in the experiment.

\section{Footnote}

Conflicts of Interest: The authors have no conflicts of interest to declare.

Ethical Statement: The authors are accountable for all aspects of the work in ensuring that questions related to the accuracy or integrity of any part of the work are appropriately investigated and resolved. This study was reviewed and approved by the experimental animal ethics committee of Wenzhou Medical University (wydw20120095) and consistent with the ethical guidelines for investigation of experimental pain in animals.

\section{References}

1. Choi DS, Choi DY, Whittington RA, et al. Sudden amnesia resulting in pain relief: The relationship between memory and pain. Pain 2007;132:206-10.

2. Bliss TV, Collingridge GL. A synaptic model of memory: long-term potentiation in the hippocampus. Nature 1993;361:31-9.

3. Sacktor TC. PKMzeta, LTP maintenance, and the dynamic molecular biology of memory storage. Prog Brain Res 2008;169:27-40.

4. Sactor TC. Memory maintenance by $\mathrm{PKM} \zeta$ - an evolutionary perspective. Mol Brain 2012;18:5-31.

5. Kwapis JL, Jarome TJ, Gilmartin MR, et al. Intraamygdala infusion of the protein kinase Mzeta inhibitor ZIP disrupts foreground context fear memory. Neurobiol Learn Mem 2012;98:148-53.

6. He YY, Xue YX, Wang JS, et al. PKM reward and aversion memory in the basolateral amygdala and extinction memory in the infralimbic cortex. Neuropsychopharmacology 2011;36:1972-81.

7. Burke NN, Geoghegan E, Kerr DM, et al. Altered neuropathic pain behaviour in a rat model of depression is associated with changes in inflammatory gene expression in the amygdala. Genes Brain Behav 2013;12:705-13.

8. Li Z, Wang J, Chen L, et al. Basolateral amygdala lesion inhibits the development of pain chronicity in neuropathic pain rats. PLoS One 2013;8:e70921.

9. Li XY, Ko HG, Chen T, et al. Alleviating neuropathic pain hyperalgesia by inhibiting $\mathrm{PKM} \zeta$ in the anterior cingulate cortex. Science 2010;330:1400-4.

10. Marchand F, D'Mello R, Yip PK, et al. Specific involvement of atypical $\mathrm{PKC} / \mathrm{PKM} \zeta$ in spinal persistent nociceptive processing following peripheral inflammation in rat. Mol Pain 2011;7:86.

11. Bennett GJ, Xie YK. A peripheral mono neuropathy in rat that produces disorders of pain sensation like those seen in man. Pain 1988;33:87-107.

12. Kwapis JL, Jarome TJ, Lonergan ME, et al. Protein kinase Mzeta maintains fear memory in the amygdala but not in the hippocampus. Behav Neurosci 2009;123:844-50.

13. Paxinos G, Watson C. The rat brain in stereotaxic coordinates, fifth ed. Amsterdam: Elsevier Academic Press, 2005.

14. Parsons RG, Gafford GM, Helmstetter FJ. Translational control via the mammalian target of rapamycin pathway is critical for the formation and stability of long-term fear memory in amygdala neurons. The Journal of Neuroscience 2006;26:12977-83.

15. Chaplan SR, Bach FW, Pogrel JW, et al. Quantitative assessment of tactile hyperalgesia in the rat paw. J Neurosci Methods 1994;53:55-63.

16. Dixon WJ. Efficient analysis of experimental observations. Annu Rev Pharmacol Toxicol 1980;20:441-62.

17. Peyron R, Laurent B, García-Larrea L. Functional imaging of brain responses to pain. Neurophysiol Clin 2000;30:263-88.

18. Gámiz F, Gallo M. Intra-amygdala ZIP injections impair the memory of learned active acquisition in rats avoidance responses and attenuate conditioned taste-aversion. Learn Mem 2011; 18:529-33.

19. Vlachos A, Maggio N, Jedlicka P. Commentary just in time for late-LTP a mechanism for the role of PKM $\zeta$ in longterm memory. Commun Integr Biol 2008;1:190-1.

20. Rainville P, Duncan GH, Price DD, et al. Pain affect encoded in human anterior cingulated but not somatosensory cortex. Science1997; 277:968-971.

21. Price TJ, Ghosh S. ZIPping to pain relief: the role (or not) of PKM $\zeta$ in chronic pain. Mol Pain 2013;9:6.

22. Sacktor TC, Fenton AA.Appropriate application of ZIP for PKM $\zeta$ inhibition, LTP reversal, and memory erasure. Hippocampus 2012;22:645-7.

23. Ikeda H, Stark J, Fischer H, et al. Synaptic Amplifier of Inflammatory Pain in the Spinal Dorsal Horn. Science 2006;312:1659-62.

Cite this article as: Yao J, Zhang L, Lei W, Huang Y, Zhang H, Lu B, Yao Y, Zhao L, Sun J. Intra-amygdala infusion of zeta inhibitory peptide attenuates neuropathic pain but not inflammatory pain in adult rats. Ann Palliat Med 2019;8(5):660666. doi: 10.21037/apm.2019.11.21 